\title{
Effect of OSW-1 on microRNA expression profiles of hepatoma cells and functions of novel microRNAs
}

\author{
JI-CHUN JIN $^{1}$, XING-LIN JIN ${ }^{1}$, XIAN ZHANG ${ }^{1}$, YING-SHI PIAO ${ }^{2,3}$ and SHUANG-PING LIU ${ }^{3,4}$ \\ ${ }^{1}$ Department of Hepatopancreatobiliary Surgery, The Affiliated Hospital of Yanbian University; \\ ${ }^{2}$ Department of Pathophysiology, Yanbian University Medical College; ${ }^{3}$ Cancer Research Center, Yanbian University; \\ ${ }^{4}$ Department of Pathology, Yanbian University Medical College, Yanji, Jilin 133000, P.R. China
}

Received December 5, 2012; Accepted April 3, 2013

DOI: $10.3892 / \mathrm{mmr} .2013 .1428$

\begin{abstract}
O-(2O-4-methoxybenzoyl- $\beta$-D-xylopyranosyl)- $(1 \rightarrow 3)$ - $(2-\mathrm{O}$-acet yl- $\alpha$-L-arabinopyranoside) (OSW-1) is a member of the cholestane saponin family, which was first isolated from the bulbs of Ornithogalum saundersiae and previously reported to be cytotoxic against several types of malignant cells. However, its antitumor mechanism remains unclear. Therefore, we investigated microRNA (miRNA) expression profiles in order to explore the antitumor activities of OSW-1. Furthermore, following study of differentially expressed miRNAs, the function of novel miRNAs and OSW-1 was determined using known miRNAs and anticarcinogens. The present study demonstrated that treatment with OSW-1 leads to the upregulation and downregulation of a large set of tumor-related miRNAs, including miR-299, miR-1908, miR-125b, miR-187a, miR-1275, hav1-miR-H6-3p, miR-181, miR-210, miR-483, miR-126, miR-208 and others. Notably, miR-141, miR-142, miR-200C and miR-1275 were found to be upregulated by OSW-1 and doxorubicine, as compared with doxorubicine alone. Additionally, the expression fold-change of miR-142-3P was $\sim 58$ times higher than its expression with a different treatment. These miRNAs are linked to cancer, including proliferation, differentiation, apoptosis, cell adhesion, migration, polarity and epithelial to mesenchymal transition (EMT).
\end{abstract}

\section{Introduction}

$3 \beta, 16 \beta, 17 \alpha$-trihydroxycholest-5-en-22-one 16-O-(2-O-4methoxybenzoyl- $\beta$-D-xylopyranosyl)- $(1 \rightarrow 3)-(2-\mathrm{O}$-acetyl- $\alpha-\mathrm{L}-$ arabinopyranoside) (OSW-1) is found in the bulbs of Ornithogalum saundersiae (1) and has high antitumor activity at

Correspondence to: Professor Xinglin Jin, Department of Hepatopancreatobiliary Surgery, The Affiliated Hospital of Yanbian University, 1327 Juzi St., Yanji, Jilin 133000, P.R. China

E-mail: xljin@ybu.edu.cn

Key words: $3 \beta, 16 \beta, 17 \alpha$-trihydroxycholest-5-en-22-one $\quad$ 16-O-(2O-4-methoxybenzoyl- $\beta$-D-xylopyranosyl)-(1 $\rightarrow 3)-(2-\mathrm{O}$-acetyl- $\alpha-\mathrm{L}$ arabinopyranoside), microRNA array, hepatoma cell, antitumor nanomolar concentrations. Its anticancer effect is 10-100 times that of doxorubicin, camptothecin and paclitaxel (2). Benign cells have been shown to be significantly less sensitive to OSW-1 compared with cancer cell lines; concentrations leading to a $50 \%$ loss in cell viability were 40-150 times greater than those required in malignant cells. Furthermore, OSW-1 may lead to the loss of mitochondrial transmembrane potential, an increase in cytosolic calcium and the activation of calcium-dependent apoptosis in human leukemia and pancreatic cancer cells (3). Although its total chemical synthesis was achieved in 1999, the anticancer activity of OSW-1 is complex and the exact mechanisms responsible for such selectivity remain unclear (4).

MicroRNAs (miRNAs) are short ( 22 nt) noncoding single-stranded RNAs that post-transcriptionally regulate protein-coded gene expression in plants and animals (5). They are able to bind to complementary sequences in the 3'-untranslated regions (3'-UTRs) of several target mRNAs to induce degradation or translational repression, and are important in cellular proliferation and development. Mature miRNAs use a silencing mode similar to that employed by siRNAs that cleave mRNA transcription $(6,7)$. Although it has been established that the human genome contains hundreds of miRNA genes and that each miRNA regulates a large number of mRNA targets, the overall effect of miRNAs on mRNA profiles has not been clarified.

To investigate the mechanism of the anticancer activity of OSW-1, we used miRNA expression analysis in hepatocellular carcinoma cells (HCCs; Hep3B) that were incubated with OSW-1 in vitro. Known differential miRNA expression was associated with the unknown function of OSW-1, and we identified the expression of unknown differential miRNAs caused by the antitumor effect of OSW-1.

\section{Materials and methods}

miRNA microarray. The 6th generation miRCURY ${ }^{\mathrm{TM}}$ LNA Array (v16.0; Exiqon, Vedbaek, Denmark) contained $>1891$ capture probes, covering all human, mouse and rat miRNAs annotated in miRBase 16.0, in addition to all the viral miRNAs associated with these species. Furthermore, the array contained capture probes for 66 new miRPlus ${ }^{\mathrm{TM}}$ human miRNAs. 
Table I. RNA quantification and quality assurance, as determined by the NanoDrop ND-1000 spectrophotometer.

\begin{tabular}{|c|c|c|c|c|c|}
\hline Group & OD260/280 ratio & OD260/230 ratio & Conc. $(\mathrm{ng} / \mu \mathrm{l})$ & Volume $(\mu 1)$ & Quantity (ng) \\
\hline A & 1.92 & 1.99 & 212.07 & 10 & 2120.70 \\
\hline B & 1.95 & 1.95 & 557.38 & 10 & 5573.80 \\
\hline $\mathrm{C}$ & 2.00 & 2.02 & 553.75 & 10 & 5537.50 \\
\hline $\mathrm{D}$ & 1.99 & 1.91 & 706.17 & 10 & 7061.70 \\
\hline
\end{tabular}

For spectrophotometry, an OD260/A280 ratio of 2.0 indicates pure RNA (ratios between 1.8-2.1 are acceptable). The OD260/A230 ratio should be $>1.8$.

Cell cultures. Hep3B cells were obtained from the Chinese Academy of Sciences Cell Bank (Shanghai, China) and the cell line was maintained in DMEM supplemented with $10 \%$ fetal bovine serum. Monoclonal cell lines were acquired using a limiting dilution assay (LDA) and maintained in DMEM with $20 \%$ fetal bovine serum. A humidified incubator was set at $37^{\circ} \mathrm{C}$ with $5 \% \mathrm{CO}_{2}$. The cell lines were divided into four groups: Group A, the Hep3B monoclonal cell line, acting as the control group; group B, monoclonal cell line treated with $200 \mathrm{ng} / \mathrm{ml} \mathrm{OSW-1} \mathrm{for} 24 \mathrm{~h}$; group C, monoclonal cell line treated with $5000 \mathrm{ng} / \mathrm{ml}$ doxorubicin for $24 \mathrm{~h}$; and group D, monoclonal cell line treated with $80 \mathrm{ng} / \mathrm{ml} \mathrm{OSW}-1$ and $2000 \mathrm{ng} / \mathrm{ml}$ doxorubicin for $24 \mathrm{~h}$. The study was approved by the ethics committee of Yanbian University, Yanji City, China.

miRNA isolation and purification. TRIzol reagent $(1 \mathrm{ml})$ (Invitrogen, Carlsbad, CA, USA) was added to a $3.5-\mathrm{cm}$ diameter dish and the cell lysate was passed through a pipette several times. The isolation and purification of miRNAs were completed using the miRNeasy Mini Kit (Qiagen, Hilden, Germany), according to the manufacturer's instructions. RNA concentration and purity were determined by measuring the absorbance at $260 \mathrm{~nm}$ and the OD260/OD280 ratio was calculated using a NanoDrop ND-1000 spectrophotometer (Nanodrop Technologies, Wilmington, DE, USA). RNA integrity and gDNA contamination were analyzed using denaturing agarose gel electrophoresis.

RNA extraction. Total RNA was isolated using TRIzol (Invitrogen) and the miRNeasy Mini Kit (Qiagen) according to the manufacturer's instructions, which recovered all RNA species, including miRNAs. RNA quality and quantity was measured using the NanoDrop spectrophotometer and RNA integrity was determined by gel electrophoresis.

RNAlabeling.AfterRNAisolation, the miRCURYHy3 $3^{\mathrm{TM}} / \mathrm{Hy} 5^{\mathrm{TM}}$ Power Labeling kit (Exiqon) was used according to the manufacturer's instructions for miRNA labeling. One microgram of each sample was 3'-end-labeled with $\mathrm{Hy} 3$ and Hy5 fluorescent labels, respectively, using T4 RNA ligase by the following procedure: RNA in $2.0 \mu \mathrm{l}$ water was combined with $1.0 \mu \mathrm{l} \mathrm{CIP}$ buffer and CIP (Exiqon). The mixture was incubated for $30 \mathrm{~min}$ at $37^{\circ} \mathrm{C}$ and terminated by incubation for $5 \mathrm{~min}$ at $95^{\circ} \mathrm{C}$. Next, $3.0 \mu \mathrm{l}$ of labeling buffer, $1.5 \mu \mathrm{l}$ of fluorescent label (Hy3 or Hy5), $2.0 \mu \mathrm{l}$ of DMSO and $2.0 \mu \mathrm{l}$ of labeling enzyme were added to the mixture. The labeling reaction was incubated for $1 \mathrm{~h}$ at $16^{\circ} \mathrm{C}$ and terminated by incubation for $15 \mathrm{~min}$ at $65^{\circ} \mathrm{C}$.

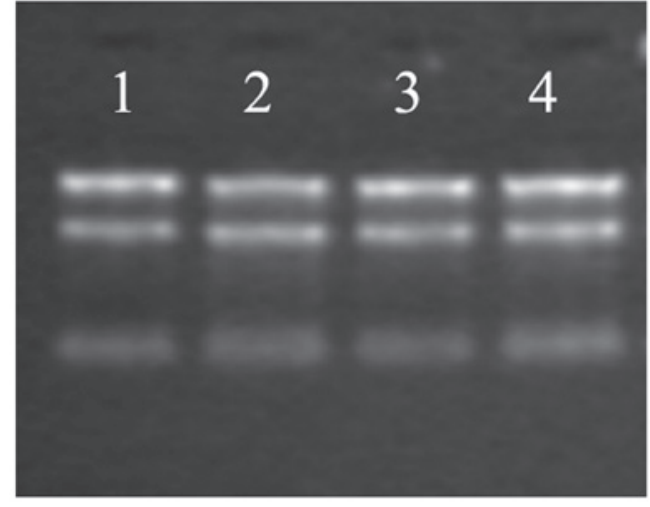

Figure 1. RNA integrity and gDNA contamination test using denaturing agarose gel electrophoresis. Lane 1, total RNA of group A; lane 2, total RNA of group B; lane 3, total RNA of group C; and lane 4, total RNA of group D. The $28 \mathrm{~S}$ and $18 \mathrm{~S}$ ribosomal RNA bands were fairly sharp, intense bands. The intensity of the upper bands were $\sim 2$ times that of the lower band. Smaller, more diffuse bands representing low-molecular weight RNAs (tRNA and $5 \mathrm{~S}$ ribosomal RNA) were present. This is likely to be comprised of mRNA and other heterogeneous RNA species. We obtained high purity mRNA and there was no DNA contamination of the RNA preparation.

Array hybridization. After terminating the labeling procedure, the Hy3- and Hy5-labeled samples were mixed pair-wise and hybridized on the miRCURY LNA Array (v16.0; Exiqon) according to the manufacturer's instructions. The total $25 \mu 1$ mixture from the Hy3- and Hy5-labeled samples with $25 \mu \mathrm{l}$ hybridization buffer was denatured for $2 \mathrm{~min}$ at $95^{\circ} \mathrm{C}$, incubated on ice for $2 \mathrm{~min}$ and then hybridized to the microarray for $16 \mathrm{~h}$ at $56^{\circ} \mathrm{C}$ in a 12-Bay Hybridization System (Nimblegen Systems Inc., Madison, WI, USA), which provided an active mixing action and constant incubation temperature to improve hybridization uniformity and enhance the signal. Following hybridization, the slides were washed several times using a wash buffer kit (Exiqon) and dried by centrifugation for $5 \mathrm{~min}$ at 400rpm.The slideswerescannedusing the Axon GenePix 4000B microarray scanner (Axon Instruments, Foster City, CA, USA).

Data analysis. Scanned images were imported into GenePix Pro 6.0 software (Axon Instruments) for grid alignment and data extraction. miRNAs with two channel intensities, $>0$ and $\mathrm{SNR}>1$, were selected for further normalization. Expression data were normalized using the locally weighted scatter plot smoothing (LOWESS) regression algorithm (8), which produced within-slide normalization to minimize the intensity-dependent differences between the dyes. Between slides normalization was performed 

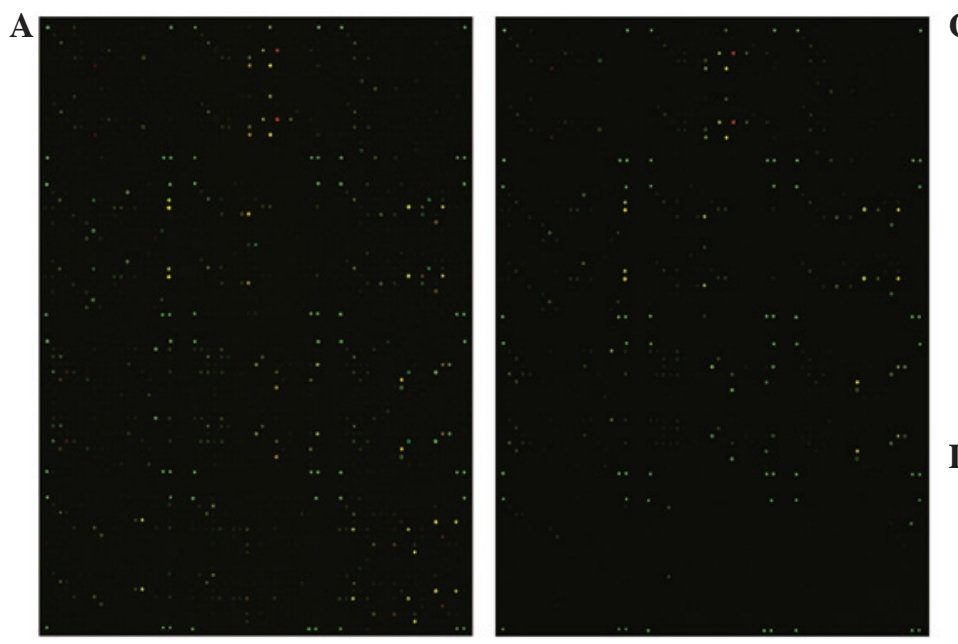

C

B
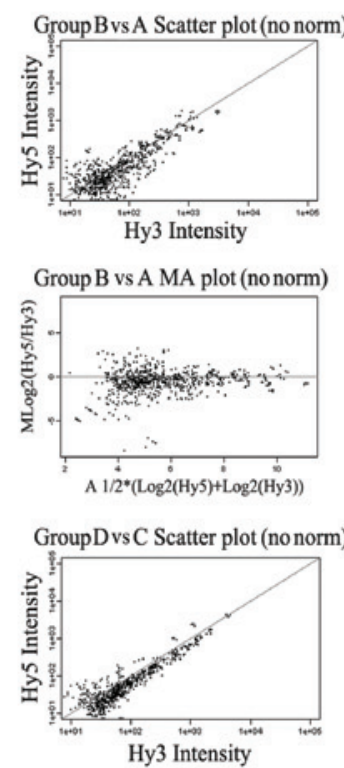

Group D C MA plot (nonorm)

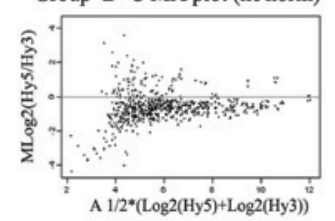

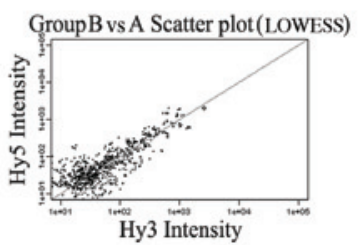

GroupBvs A MA plot (nonorm)

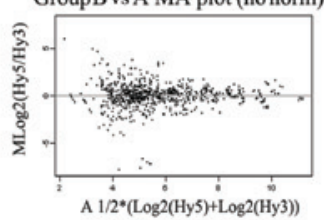

GroupDvsC Scatter plot (LOWESs)
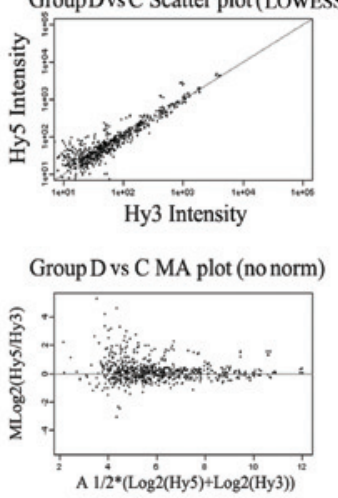
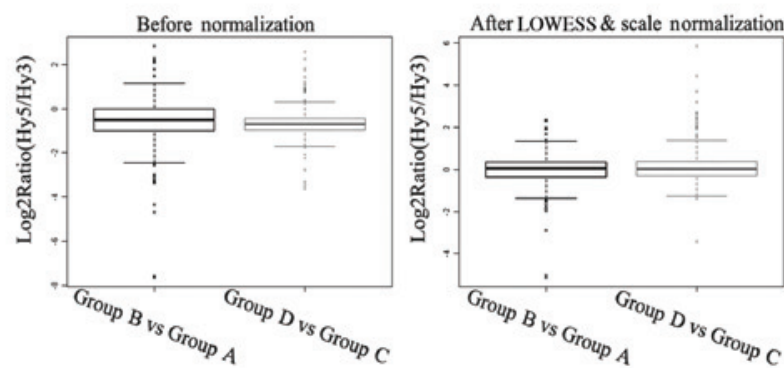

D
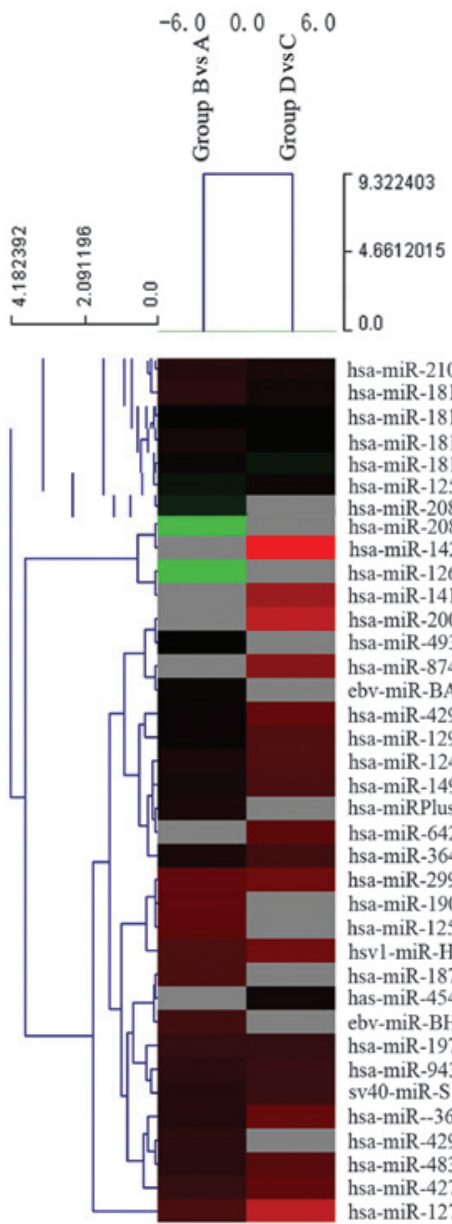

hsa-miR-210 hsa-miR-181-5P hsa-miR-181a-2-3p hsa-miR-181d hsa-miR-181b-5p hsa-miR-125b-sp hsa-miR-208a hsa-miR-142-3p hsa-mik-142-3p hsa-miR-14l-3p hsa-miR-141-3p hsa-miR-200c-3p hsa-miR-493-5p hsa-miR-874 ebv-miR-BART1 hsa-miR-4290 hsa-miR-1290 hsa-miR-1246 hsa-miR-149-3P hsa-miRPlus-C1110 hsa-miR-642a-5p hsa-miR-3646 hsa-miR-299-5p hsa-miR-1908 hsa-miR-125b-1-3p hsa-miR-187-5p has-miR-454-3p ebv-miR-BHRF1-2 hsa-miR-1973 hsa-miR-943 sv40-miR-S1-5p hsa-miR--3611 hsa-miR-4299 hsa-miR-483-3p hsa-miR-4279 hsa-miR-1275

Figure 2. (A). Graphs scanned from slides using a microarray scanner. These graphs were imported into GenePix Pro 6.0 software for grid alignment and data extraction. (B) The MA-plot shows the distribution of the red/green intensity ratio ('M') plotted by the average intensity ('A'). M and A are defined by the following equations: $M=\log _{2} R-\log _{2} G$ and $A=1 / 2 \times\left(\log _{2} R+\log _{2} G\right)$, where $R$ and $G$ represent the probe raw signal intensity for the MeDIP and Input channels, respectively. After applying LOWESS normalization, the two samples hybridized on the array may show balanced differences with the M-values distributed around zero. (C) The box plots are a convenient way to quickly visualize the distribution of a dataset and are most useful for comparing the distributions of samples. After normalization, the distributions of $\log _{2}$-ratios across every sample are almost the same. (Left, non-normalized $\log _{2}$-ratio data; right, LOWESS and scale normalized $\log _{2}$-ratio data). (D) A section of the heat map diagram demonstrating the result of the two-way hierarchical clustering of microRNAs (miRNAs) and groups. Each row represents a single miRNA and each column represents a group. The miRNA clustering tree is shown on the left and the sample clustering tree appears at the top. This hierarchical clustering was performed based on the ratio of miRNAs. The result of hierarchical clustering shows distinguishable miRNA expression profiling among groups. Red indicates high relative expression and green indicates low relative expression. LOWESS, locally weighted scatter plot smoothing regression algorithm.

using scale normalization (9). Following normalization, replicated miRNAs were averaged. Differentially expressed miRNAs were identified using fold-change filtering. Hierarchical clustering was performed using MEV software (v4.6, TIGR) (10). Quantitative RT-PCR verified the miRNA expression profile using random sampling.

\section{Results}

Eligible total RNA was isolated, including miRNAs, for the miRNA microarray from the cell cultures, as shown in Table I and Fig. 1. Scanned images were imported into GenePix Pro 6.0 software for grid alignment and 
Table II. miRNA expression profiling data.

\begin{tabular}{|c|c|c|c|c|c|c|c|c|}
\hline \multirow[b]{3}{*}{ ID } & \multirow[b]{3}{*}{ Name } & \multirow{2}{*}{\multicolumn{2}{|c|}{ Fold-change }} & \multirow{2}{*}{\multicolumn{2}{|c|}{$\begin{array}{c}\begin{array}{c}\text { Data after LOWESS } \\
\text { normalization }\end{array} \\
\log _{2} \text { ratio scale }\end{array}$}} & \multicolumn{3}{|c|}{ Data after LOWESS \& scale normalization } \\
\hline & & & & & & \multicolumn{2}{|c|}{$\log _{2}$ ratio scale } & \multirow{2}{*}{$\begin{array}{c}\text { Ratio scale } \\
\begin{array}{c}\text { Group B/ } \\
\text { group A }\end{array}\end{array}$} \\
\hline & & $\begin{array}{c}\text { Group B/ } \\
\text { group A }\end{array}$ & $\begin{array}{l}\text { Group D/ } \\
\text { group C }\end{array}$ & $\begin{array}{c}\text { Group B/ } \\
\text { group A }\end{array}$ & $\begin{array}{l}\text { Group D/ } \\
\text { group C }\end{array}$ & $\begin{array}{c}\text { Group B/ } \\
\text { group A }\end{array}$ & $\begin{array}{l}\text { Group D/ } \\
\text { group C }\end{array}$ & \\
\hline 4040 & hsa-miR-9-5p & 1.201596352 & 0.973057734 & 0.376576901 & -0.027722981 & 0.264952338 & -0.039402688 & 1.201596352 \\
\hline 4610 & hsa-miR-126-3p & 0.030305072 & & -7.169461969 & & -5.044296936 & & 0.030305072 \\
\hline 4700 & hsa-miR-140-5p & 1.73666148 & 1.47424504 & 1.131805162 & 0.393988691 & 0.796316563 & 0.55997634 & 1.73666148 \\
\hline 5250 & hsa-miR-105-5p & & & & & & & \\
\hline 5730 & hsa-miR-208a & 0.02847763 & & -7.296995474 & & -5.134027082 & & 0.02847763 \\
\hline 6880 & hsa-miR-297 & & & & & & & \\
\hline 9938 & hsa-let-7i-5p & 0.769523042 & 0.641605265 & -0.537199825 & -0.450462161 & -0.377963568 & -0.640242114 & 0.769523042 \\
\hline 10138 & hsa-miR-130a-3p & 1.056391584 & 1.164773916 & 0.112488423 & 0.154822957 & 0.079144713 & 0.220049953 & 1.056391584 \\
\hline 10306 & hsa-miR-146b-5p & & & & & & & \\
\hline 10916 & hsa-miR-1 & & & & & & & \\
\hline 10919 & hsa-miR-103a-3p & 1.130481377 & 1.167560403 & 0.251480975 & 0.157248367 & 0.176937226 & 0.223497189 & 1.130481377 \\
\hline 10923 & hsa-miR-107 & 0.920825852 & 0.856509091 & -0.169134419 & -0.157221875 & -0.118999757 & -0.223459536 & 0.920825852 \\
\hline 10925 & hsa-miR-10b-5p & 1.345927976 & 0.489139642 & 0.609171131 & -0.725871604 & 0.428601209 & -1.031681704 & 1.345927976 \\
\hline 10928 & hsa-miR-125a-5p & 1.260412915 & 1.311638016 & 0.474567194 & 0.275360214 & 0.333896442 & 0.391369622 & 1.260412915 \\
\hline
\end{tabular}

List only represents part of the whole results. ID, the miRNA ID number constituted by Exiqon; name, miRNA name; normalized value, shows normalized log 2 ratio scale or normalized ratio scale; LOWESS, locally weighted scatter plot smoothing regression algorithm.

A

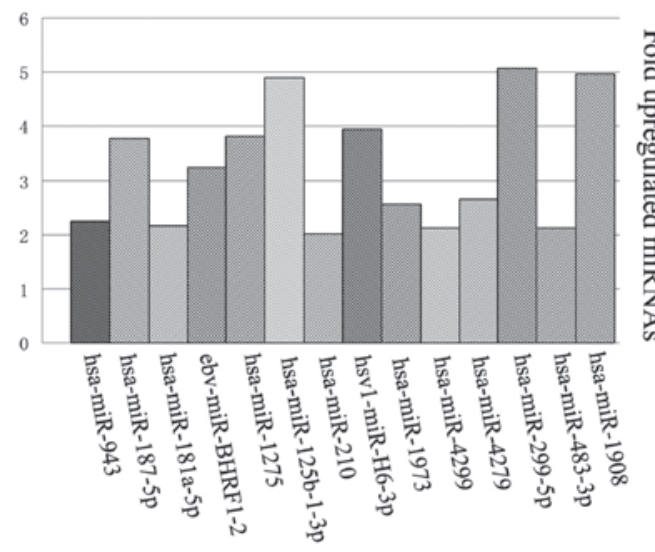

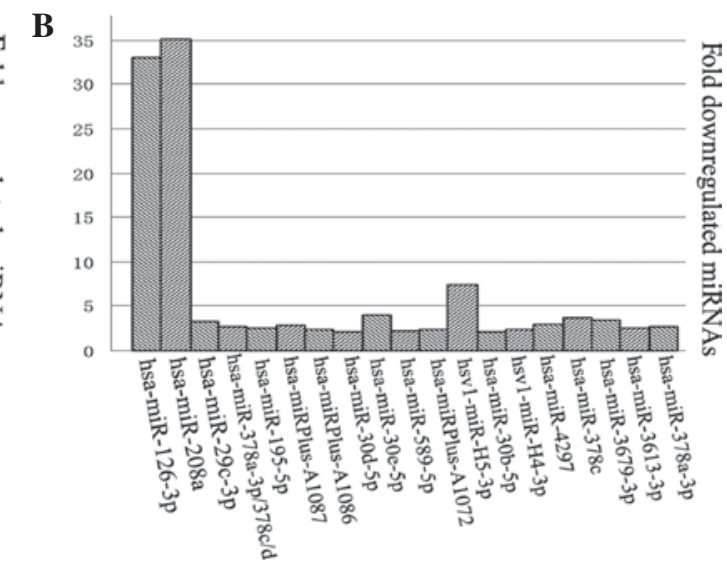

Figure 3. Levels of upregulation and downregulation in the OSW-1 group compared with those of the control group (group B vs group A) for differentially

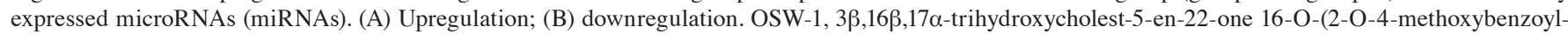
$\beta$-D-xylopyranosyl)-(1 $\rightarrow 3)$ - (2-O-acetyl- $\alpha$-L-arabinopyranoside).

data extraction (Fig. 2A). miRNAs with two channel intensities, $>0$ and $\mathrm{SNR}>1$, were selected for further normalization. Expression data were normalized using the LOWESS regression algorithm (8), which produced withinslide normalization to minimize the intensity-dependent differences between the dyes. Between slides normalization was performed by scale normalization (Table II) (9) - After normalization, the replicated miRNAs were averaged. Differentially expressed miRNAs were identified through fold-change filtering and quality assessments of miRNA data were performed after filtering, as shown in Fig. 2B and C and Table III. Finally, hierarchical clustering was carried out to reveal distinguishable miRNA expression profiling among the four groups (Fig. 2D).

Treatment with OSW-1 induced the upregulation of a number of miRNAs, including miR-181, miR-187, miR-210, miR-2, miR-299 and miR-1275 (Fig. 3A). Notably, the downregulation of miRNAs also occurred with OSW-1 treatment, to include miR-126 and miR-208. Following 24 h of OWS-1 treatment, there was an $\sim 35$-fold downregulation of miRNA (Fig. 3B).

The effect of OSW-1 was compared with that of traditional anticarcinogens through identification of the differentially expressed miRNAs among group D and group C. Results showed that miR-1275, miR-200 and miR-141 were upregulated 
Table III. All differentially expressed miRNAs.

A, Group B/group A 2.0-fold upregulated miRNAs

\begin{tabular}{|c|c|c|c|c|c|c|c|}
\hline \multirow[b]{2}{*}{ ID } & \multirow[b]{2}{*}{ Name } & \multirow{2}{*}{ 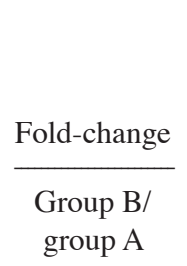 } & \multicolumn{2}{|c|}{ Raw intensity } & \multirow{2}{*}{$\begin{array}{c}\log _{2} \text { ratio scale } \\
\begin{array}{c}\text { after LOWESS } \\
\text { normalization }\end{array} \\
\begin{array}{c}\text { Group B/ } \\
\text { group A }\end{array}\end{array}$} & \multirow{2}{*}{$\begin{array}{c}\log _{2} \text { ratio scale } \\
\text { after LOWESS } \\
\text { and scale } \\
\text { normalization } \\
\text { Group B/ } \\
\text { group A }\end{array}$} & \multirow{2}{*}{$\begin{array}{c}\begin{array}{c}\text { Ratio scale after } \\
\text { LOWESS } \\
\text { and scale } \\
\text { normalization }\end{array} \\
\begin{array}{c}\text { Group B/ } \\
\text { group A }\end{array}\end{array}$} \\
\hline & & & $\begin{array}{c}\text { Group B } \\
(\mathrm{Hy} 5)\end{array}$ & $\begin{array}{c}\text { Group A } \\
\text { (Hy3) }\end{array}$ & & & \\
\hline 42696 & hsa-miR-943 & 2.243215941 & 26.5 & 13.5 & 1.65662315 & 1.165568507 & 2.243215941 \\
\hline 42775 & hsa-miR-187-5p & 3.766473652 & 33.0 & 9.5 & 2.719252716 & 1.913214437 & 3.766473652 \\
\hline
\end{tabular}

B, Group B/group A 1 2.0-fold downregulated miRNAs

\begin{tabular}{|c|c|c|c|c|c|c|c|}
\hline \multirow[b]{2}{*}{ ID } & \multirow[b]{2}{*}{ Name } & \multirow{2}{*}{$\begin{array}{c}\text { Fold-change } \\
\begin{array}{c}\text { Group B/ } \\
\text { group A }\end{array}\end{array}$} & \multicolumn{2}{|c|}{ Raw intensity } & \multirow{2}{*}{ 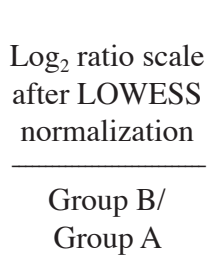 } & \multirow{2}{*}{ 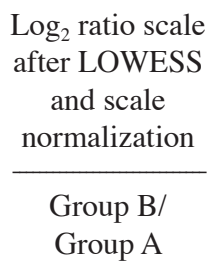 } & \multirow{2}{*}{$\begin{array}{c}\begin{array}{c}\text { Ratio scale after } \\
\text { LOWESS } \\
\text { and scale } \\
\text { normalization }\end{array} \\
\begin{array}{c}\text { Group B/ } \\
\text { Group A }\end{array}\end{array}$} \\
\hline & & & $\begin{array}{c}\text { Group B } \\
\text { (Hy5) }\end{array}$ & $\begin{array}{c}\text { Group A } \\
(\mathrm{Hy} 3)\end{array}$ & & & \\
\hline 4610 & hsa-miR-126-3p & 0.030305072 & 0 & 378 & -7.169461969 & -5.044296936 & 0.030305072 \\
\hline 5730 & hsa-miR-208a & 0.02847763 & 3 & 572 & -7.296995474 & -5.134027082 & 0.02847763 \\
\hline
\end{tabular}

Only represents part of differentially expressed miRNAs after fold-change filtering. ID, the miRNA ID number constituted by Exiqon; name, miRNA name; fold-change, the ratio scale; raw intensity, raw intensities of samples in the Hy 5 or Hy 3 channel; ratio scale/log ${ }_{2}$ ratio scale, ratio scale/log 2 ratio scale values after normalization; LOWESS, locally weighted scatter plot smoothing regression algorithm.

13 to 21-fold. Notably, the level of upregulation of miR-142 was $~ 58$-fold higher than its expression with a different treatment (Fig. 4A and B).

\section{Discussion}

miRNAs, including hsa-miR-299-5p, miR-125b, miR-187-5p and miR-210, were upregulated following treatment with OSW-1. In a study by Shevde et al (11), hsa-mir-299-5p targeted osteopontin (OPN), which is known to be critical for enhancing proliferation and tumorigenicity. We speculate that the expression of OPN is downregulated following treatment with OSW-1, thus inhibiting the proliferation and tumorigenicity of HCCs. miR-125b has been shown to suppress HCC proliferation and metastasis by directly targeting the oncogene LIN28B (12), the overexpression of which represses the endogenous level of the p53 protein, thus suppressing apoptosis in human neuroblastoma and lung fibroblast cells (13). In accordance with our previous study (14), the p53 pathway was activated by OSW-1 on the miRNA level. miR-187 is highly expressed in $B R A F$ and $R A S$ point mutations and RET/PTC and PAX8/PPAR rearrangements (15). It has been demonstrated that OSW-1 acts directly on $B R A F$ mutations to highly express miR-187. miR-210 mediates a novel mechanism of adaptation to hypoxia and regulates mitochondrial function via iron-sulfur cluster metabolism and free radical generation (16). The balance of the reactive oxygen species (ROS) is important for maintaining a normal metabolism. In previous studies, malignant cells were transformed by oxidative damage to DNA and mutations were induced by the sustained activation of ROS. ROS activates the p53 signaling pathway, influencing mitochondrial membrane potential and promoting the generation of caspase-3, caspase- 9 , and representative drugs, doxorubicin and vincristine $(17,18)$. Furthermore, high levels of miR-483-3p may be found in $30 \%$ of colon, breast and liver cancers, which is located within intron 2 of the IGF2 locus (19). Our data showed that miR-483 was highly expressed after treatment with OSW-1, which indicates that the function of miR-483 was bidirectional.

hsa-miR-126-3p and hsa-miR-208a were downregulated $\sim 58$-fold by OSW-1. miR-126 is known to enhance the pro-angiogenic actions of VEGF and FGF and promote blood vessel formation by repressing the expression of Spred-1 accordingly. The targeted deletion of miR-126 in mice produced leaking vessels, hemorrhaging and partial embryonic lethality (20). After treatment with OSW-1, the expression levels of miR-126 in HCCs were barely detected, which may increase the ability of Spred-1 to inhibit RAF. Tumor angiogenesis was prohibited through the inhibition of the MAPK signaling pathway by OSW-1. Therefore, the proliferation of vascular smooth muscle cells correlates with miR-208-mediated downregulation of p21 (21), demonstrating that OSW-1 may inhibit tumor growth by the vasifaction of tumor tissue.

Comparison between group $\mathrm{C}$ and group $\mathrm{D}$ revealed that miR-142 was expressed at high levels, and miR-200C-3p, miR-1275 and miR-141 showed increasing levels of upregulation from 13 to 20-fold (Fig. 4). miR-142-3p directly and negatively regulates RAC1 in HCCs, where RAC1 regulates a diverse array of cellular events including migration and 
A

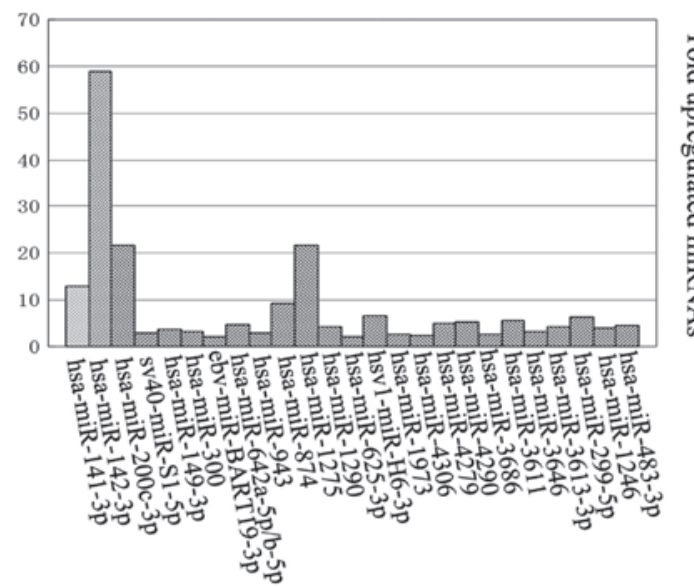

B

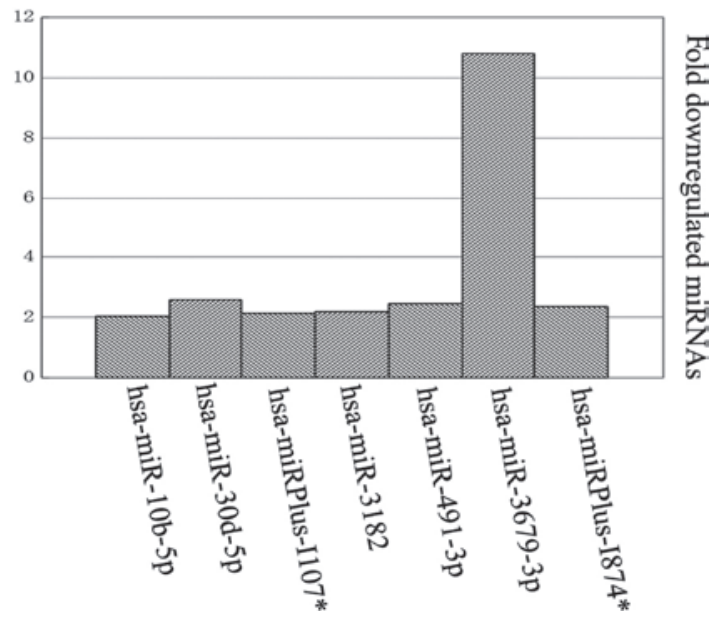

Figure 4. Levels of upregulation and downregulation in the OSW-1 and doxorubicin group compared with those of the doxorubicin group (group D vs group C) for differentially expressed microRNAs (miRNAs). (A)Upregulation;(B)downregulation.OSW-1,3 $\beta, 16 \beta, 17 \alpha$-trihydroxycholest-5-en22-one 16-O-(2-O-4-methoxybenzoyl- $\beta$-D-xylopyranosyl)-(1 $\rightarrow 3)$ - (2-O-acet yl- $\alpha$-L-arabinopyranoside).

invasion (22). In a study by Wu et al (22), blocking miR-142-3p increased colony formation, migration and invasion in HCCs. The levels of miR-142 were inversely related to the levels of acetyltransferase p300 and MAPK activity, and miR-142 inhibited survival and growth pathways by directly targeting nodal regulators $\mathrm{p} 300$ and gp130. miR-142 potentially represses multiple components of the NF- $\mathrm{NB}$ pathway, preventing cytokine-mediated NO production and blocking the translation of actinin (23). The upregulation of miR-142 by OSW-1 inhibits RAC1 in the Wnt, MAPK and axon guidance pathway. Overexpression of miR-200c leads to reduced levels of transcription factor 8 and increased expression of E-cadherin (24). OSW-1-mediated upregulation of miR-200c enhances the intercellular junctions of HCCs by stabilizing cell polarity and inhibiting proliferation. Simultaneously, miR-141 and miR-200c inhibit the expression of ZFHX1B, a transcriptional repressor for $\mathrm{CDH} 1 / \mathrm{E}$-cadherin (25). Zinc finger E-box-binding homeobox 1 (ZEB1) and ZEB2 are regulated by the high expression of the miR-200 family, which weakens the inhibition of E-cadherin, preventing the occurrence of epithelial to mesenchymal transition (EMT) (26).

Under the influence of OSW-1, the upregulated expression of miR-1275 was increased 4-fold, and following treatment

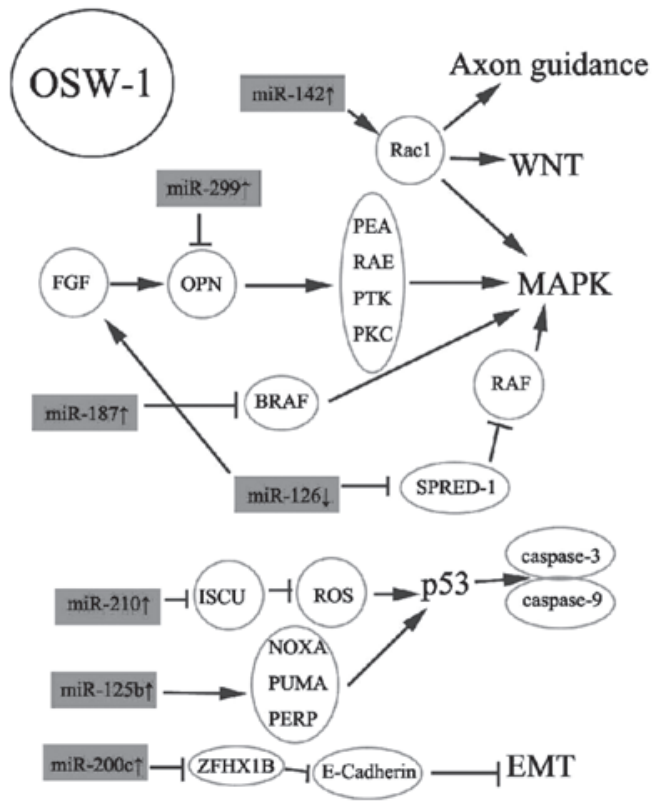

Figure 5. OSW-1 may affect numerous microRNAs that act on specific signaling pathways for proliferation, differentiation, apoptosis, cell adhesion, migration, polarity and mesenchymal transition (EMT). OSW-1, 3 $\beta, 16 \beta, 17 \alpha$-trihydroxycholest-5-en-22-one 16-O-(2-O-4-methoxybenzoyl- $\beta$-D-xylopyranosyl)-( $1 \rightarrow 3)$ - (2-O-acetyl- $\alpha-\mathrm{L}$ arabinopyranoside); ROS, reactive oxygen species.

with OSW-1 and doxorubicin, it was upregulated with an 20-fold increase when compared to the effects of doxorubicin alone. We hypothesized that miR-1275 is a direct target of OSW-1 and therefore is a potential anticancer target.

In conclusion, OSW-1 may affect numerous miRNAs that act on specific signaling pathways for proliferation, differentiation, apoptosis, cell adhesion, migration, polarity and EMT (Fig. 5).

\section{Acknowledgements}

This study was supported by a grant from the National Natural Science Foundation of China (Grant No. 81160529) and the Jilin province Science and Technology Development Project (Grant No. 200905207).

\section{References}

1. Kubo S, Mimaki Y, Terao M, Sashida Y, Nikaido T and Ohmoto T: Acylated cholestane glycosides from the bulbs of Ornithogalum saudersiae. Phytochemistry 31: 3969-3973, 1992.

2. Mimaki Y, Kuroda M, Kameyama A, Sashida Y, Hirano T, and Oka K: Cholestane glycosides with potent cytostatic activities on various tumor cells from Ornithogalum saundersiae bulbs. Bioorg Med Chem Lett 7: 633-636, 1997.

3. Zhou Y, Garcia-Prieto C, Carney DA, et al: OSW-1: a natural compound with potent anticancer activity and a novel mechanism of action. J Natl Cancer Inst 97: 1781-1785, 2005.

4. Deng S, Yu B, Lou Y and Hui Y: First total synthesis of an exceptionally potent antitumor saponin, OSW-1. J Org Chem 64: 202-208, 1999.

5. Morris KV, Chan SW, Jacobsen SE and Looney DJ: Small interfering RNA-induced transcriptional gene silencing in human cells. Science 305: 1289-1292, 2004

6. Jeyaseelan K, Herath WB and Armugam A: MicroRNAs as therapeutic targets in human diseases. Expert Opin Ther Targets 11: 1119-1129, 2007.

7. Scherr M and Eder M: Gene silencing by small regulatory RNAs in mammalian cells. Cell Cycle 6: 444-449, 2007. 
8. Cleveland WS: LOWESS: A program for smoothing scatterplots by robust locally weighted regression. Am Stat 35: 54, 1981.

9. Yang YH, Dudoit S, Luu P, Lin DM, Peng V, Ngai J and Speed TP: Normalization for cDNA microarray data: a robust composite method addressing single and multiple slide systematic variation. Nucleic Acids Res 30: e15, 2002.

10. Saeed AI, Bhagabati NK, Braisted JC, Liang W, Sharov V, Howe EA, et al: TM4 microarray software suite. Methods in Enzymology 411: 134-193, 2006.

11. Shevde LA, Metge BJ, Mitra A, Xi Y, Ju J, King JA and Samant RS: Spheroid-forming subpopulation of breast cancer cells demonstrates vasculogenic mimicry via hsa-miR-299-5p regulated de novo expression of osteopontin. J Cell Mol Med 14 1693-1706, 2010

12. Liang L, Wong CM, Ying Q, et al: MicroRNA-125b suppressesed human liver cancer cell proliferation and metastasis by directly targeting oncogene LIN28B2. Hepatology 52: 1731-1740, 2010.

13. Le MT, Teh C, Shyh-Chang N, et al: MicroRNA-125b is a novel negative regulator of p53. Genes Dev 23: 862-876, 2009.

14. Jin J, Jin X, Qian C, Ruan Y and Jiang H: Signaling network of OSW 1 induced apoptosis and necroptosis in hepatocellular carcinoma. Mol Med Rep 7: 1646-1650, 2013.

15. Nikiforova MN, Tseng GC, Steward D, Diorio D and Nikiforov YE: MicroRNA expression profiling of thyroid tumors: biological significance and diagnostic utility. J Clin Endocrinol Metab 93: 1600-1608, 2008.

16. Favaro E, Ramachandran A, McCormick R, et al: MicroRNA-210 regulates mitochondrial free radical response to hypoxia and krebs cycle in cancer cells by targeting iron sulfur cluster protein ISCU. PLoS One 5: e10345, 2010.

17. Mizutani H, Tada-Oikawa S, Hiraku Y, Kojima M and Kawanishi S: Mechanism of apoptosis induced by doxorubicin through the generation of hydrogen peroxide. Life Sci 76: $1439-1453,2005$
18. Groninger E, Meeuwsen-De Boer GJ, De Graaf SS, Kamps WA and De Bont ES: Vincristine induced apoptosis in acute lymphoblastic leukaemia cells: a mitochondrial controlled pathway regulated by reactive oxygen species? Int J Oncol 21: 1339-1345, 2002.

19. Veronese A, Lupini L, Consiglio J, et al: Oncogenic role of miR-483-3p at the IGF2/483 locus. Cancer Res 70: 3140-3149, 2010.

20. Wang S, Aurora AB, Johnson BA, et al: The endothelial-specific microRNA miR-126 governs vascular integrity and angiogenesis. Dev Cell 15: 261-271, 2008.

21. Zhang Y, Wang Y, Wang X, et al: Insulin promotes vascular smooth muscle cell proliferation via microRNA-208-mediated downregulation of p21. J Hypertens 29: 1560-1568, 2011.

22. Wu L, Cai C, Wang X, Liu M,Li X and Tang H: MicroRNA-142-3p, a new regulator of $\mathrm{RAC1}$, suppresses the migration and invasion of hepatocellular carcinoma cells. FEBS Lett 585: 1322-1330, 2011.

23. Sharma S, Liu J, Wei J, Yuan H, Zhang T and Bishopric NH: Repression of miR-142 by $\mathrm{p} 300$ and MAPK is required for survival signalling via gp130 during adaptive hypertrophy. EMBO Mol Med 4: 617-632, 2012.

24. Hurteau GJ,Carlson JA,Spivack SD and Brock GJ: Overexpression of the microRNA hsa-miR-200c leads to reduced expression of transcription factor 8 and increased expression of E-cadherin. Cancer Res 67: 7972-7976, 2007.

25. Nakada C, Matsuura K, Tsukamoto Y, et al: Genome-wide microRNA expression profiling in renal cell carcinoma: significant down-regulation of miR-141 and miR-200c. J Pathol 216: 418-427, 2008.

26. Korpal M,Lee ES, Hu G and Kang Y: The miR-200 family inhibits epithelial-mesenchymal transition and cancer cell migration by direct targeting of E-cadherin transcriptional repressors ZEB1 and ZEB2. J Biol Chem 283: 14910-14914, 2008. 